\title{
AVALIAÇÃO DAS CONDIÇÕES DE EXTRAÇÃO DA CERA OBTIDA A PARTIR DA CASCA DA CANA DE AÇÚCAR UTILIZANDO PLANEJAMENTO DO TIPO COMPOSTO CENTRAL
}

\author{
M. A. B. ESTEVES e P. F. M. MARTINEZ \\ Universidade Estadual de Campinas, Faculdade de Engenharia Química \\ E-mail para contato: M140833@dac.unicamp.br
}

\begin{abstract}
RESUMO - O Brasil é o maior produtor mundial de cana de açúcar. Consequentemente, o processamento desta matéria-prima gera elevada quantidade de subprodutos, como a casca da cana de açúcar, que é subutilizada como adubo orgânico nos campos de cultivo. Entretanto, a partir deste resíduo agroindustrial pode-se extrair cera promovendo-se assim, a valoração deste material. Dessa forma, neste trabalho avaliou-se as condições de extração da cera da cana de açúcar a fim de que haja maiores rendimentos (R) com menores custos operacionais. Para isso, analisou-se a influência do hidromódulo, tamanho de partícula, tempo e suas respectivas interações no processo de extração da cera da cana de açúcar por meio do planejamento experimental do tipo composto central. A extração foi realizada em extrator do tipo Soxhlet com hexano e os resultados dos ensaios foram tratados através do software STATISTICA 7.0. Verificou-se que o único fator de influência no nível de confiança de $99 \%$ e na faixa de estudo das condições de extração é o tamanho de partícula (X2). A diminuição do tamanho da partícula proporciona maiores rendimentos de cera bruta
\end{abstract}

\section{INTRODUÇÃO}

O Brasil é o maior produtor mundial de cana de açúcar (Saccharum officinarum), sendo este, um dos destaques do agronegócio nacional. Para a safra de 2016/17, estima-se uma produção de 694,54 milhões de toneladas, da qual, o estado de São Paulo representa o maior percentual produtivo (CONAB, 2016).

Com o processamento de grande volume dessa matéria-prima, há elevada geração de subprodutos, como a casca da cana de açúcar proveniente da preparação da cana para produção da garapa. Antes de ser enviada aos garapeiros, a cana passa por uma etapa de raspagem, em que a casca é retirada e posteriormente passa a ser subutilizada como adubo orgânico nos campos de cultivo. Assim, a investigação da extração da cera a partir desse subproduto representa uma das oportunidades para promover a valoração dos resíduos da indústria sucroalcooleira.

Diante deste cenário, o Brasil poderia ser um potencial fornecedor da cera da cana de açúcar, que pode ser utilizada para a substituição de outras ceras comerciais, como a de carnaúba, por apresentar características químico-físicas semelhantes (Nuissier et al., 2002). As ceras naturais tem aplicabilidade nas indústrias têxtil, de papéis e embalagens, alimentícia, 
química, farmacêutica, na medicina, na fabricação de cosméticos, na produção de tintas, no setor de polimento, e na restauração de pinturas em telas e modelagem (Granda, 2006).

Portanto, o estudo das condições de extração do produto torna-se de grande importância e interesse industrial. Todavia, segundo Barros Neto et al. (2001), um empecilho ao se realizar experimentos é analisar a influência das interações variando todos os fatores ao mesmo tempo.

Logo, o estudo objetiva avaliar os efeitos dos fatores de extração como, hidromódulo (razão alimentação:solvente), tamanho de partícula e tempo, em três diferentes níveis, com suas respectivas interações no processo através do planejamento experimental do tipo composto central, para que se possa avaliar as melhores condições para a extração desta cera.

\section{MATERIAIS E MÉTODOS}

\subsection{Matéria-Prima}

A casca da cana de açúcar de variedade RB 966928 foi cedida pelo Sítio Santo Antônio (Rio Claro - SP). Esta foi pulverizada em moinho elétrico do tipo martelo (Tigre S.A, CV2, Brasil), acoplado a um motor de indução de 3800 rpm (General Eletric, CV5, Brasil). O pó obtido foi classificado por tamanho de partícula utilizando equipamento de granulometria com os seguintes jogos de tamises (GRANUTEST) da série Tyler: 24, 32, 42, 48, 80, 170, 250 e 400.

\subsection{Extração da Cera da Cana de Açúcar}

A extração da cera obtida a partir da casca da cana de açúcar, com cerca de $8 \%$ de umidade, foi realizada em extrator do tipo Soxhlet com hexano utilizando-se diferentes condições operacionais de tempo, tamanho de partícula e hidromódulo. A separação entre produto e solvente se sucedeu em evaporador rotativo (TE-211 - TECNAL) e o rendimento da extração foi determinado gravimetricamente em balança eletrônica analítica (ATX 224 SHIMADZU). Para o cálculo do rendimento da cera bruta (R) empregou-se a Equação 1.

$$
\mathrm{R}(\%)=\frac{\text { Massa de cera bruta obtida }(\mathrm{g})}{\text { Massa da casca empregada na extração }(\mathrm{g})} \times 100
$$

\subsection{Planejamento Experimental}

Para determinação das melhores condições do processo de extração da cera da cana de açúcar, utilizou-se o planejamento experimental do tipo composto central, abrangendo 3 fatores, sendo eles: hidromódulo (X1), também chamado de razão alimentação:solvente, tamanho de partícula (X2) e tempo (X3), em 3 níveis distintos e 4 pontos centrais para aferição do erro experimental. A variável resposta estudada foi o rendimento de cera. Os ensaios foram realizados de maneira aleatória para que se evitassem distorções estatísticas e o tratamento dos resultados foi realizado através do software STATISTICA 7.0, com nível de confiança de 99\%. Os níveis utilizados para cada fator estão descritos na Tabela 1. 
Tabela 1 - Níveis dos fatores empregados no planejamento experimental do tipo composto central para estudo das condições de extração da cera da cana de açúcar

\begin{tabular}{cccccc}
\hline Fatores & $-\boldsymbol{\alpha}$ & $\mathbf{- 1}$ & $\mathbf{0}$ & $\mathbf{1}$ & $\mathbf{+ \alpha}$ \\
\hline Hidromódulo $(\mathrm{g} / \mathrm{ml})$ & $10: 267$ & $10: 250$ & $10: 225$ & $10: 200$ & $10: 183$ \\
Tamanho $(\mathbf{m m})$ & 0,05 & 0,13 & 0,26 & 0,39 & 0,50 \\
Tempo $(\mathrm{h})$ & 4,64 & 6 & 8 & 10 & 11,36 \\
\hline
\end{tabular}

\section{RESULTADOS E DISCUSSÃO}

A matriz do planejamento experimental, bem como os rendimentos de cera obtidos em cada ensaio são apresentados na Tabela 2. Observa-se que, a depender das condições operacionais utilizadas durante as extrações, o rendimento de cera pode variar de $4 \%$ a $16 \%$.

Tabela 2 - Matriz codificada e rendimento do planejamento do tipo composto central para estudo das condições de extração da cera da cana de açúcar

\begin{tabular}{|c|c|c|c|c|}
\hline \multirow[t]{2}{*}{ Ensaio } & \multicolumn{3}{|c|}{ Variáveis codificadas } & \multirow{2}{*}{$\begin{array}{c}\text { Rendimento } \\
(\%)\end{array}$} \\
\hline & $\mathbf{X} 1$ & $\mathbf{X} 2$ & $\mathbf{X 3}$ & \\
\hline 1 & -1 & -1 & -1 & 9,748 \\
\hline 2 & -1 & -1 & 1 & 9,713 \\
\hline 3 & -1 & 1 & -1 & 4,207 \\
\hline 4 & -1 & 1 & 1 & 4,409 \\
\hline 5 & 1 & -1 & -1 & 9,815 \\
\hline 6 & 1 & -1 & 1 & 10,670 \\
\hline 7 & 1 & 1 & -1 & 4,002 \\
\hline 8 & 1 & 1 & 1 & 4,467 \\
\hline 9 & 0 & 0 & 0 & 6,473 \\
\hline 10 & 0 & 0 & 0 & 6,581 \\
\hline 11 & 0 & 0 & 0 & 6,708 \\
\hline 12 & 0 & 0 & 0 & 6,394 \\
\hline 13 & $-1,68$ & 0 & 0 & 6,959 \\
\hline 14 & $+1,68$ & 0 & 0 & 6,677 \\
\hline 15 & 0 & $-1,68$ & 0 & 16,440 \\
\hline 16 & 0 & $+1,68$ & 0 & 4,046 \\
\hline 17 & 0 & 0 & $-1,68$ & 6,755 \\
\hline 18 & 0 & 0 & $+1,68$ & 7,004 \\
\hline
\end{tabular}

Para investigar como tais condições influenciam o rendimento de cera, faz-se imprescindível a escolha do nível de confiança e, para isso, utilizou-se o Gráfico de Probabilidades, Figura 1. Constatou-se que, exceto o efeito do tamanho de partícula, tanto linear quanto quadrática, os demais pontos, referentes aos efeitos dos fatores hidromódulo e do tempo, assim como todas as interações, se ajustam em uma reta sobre a origem, dando indícios de que estes devem ter vindo de uma distribuição normal com média zero e, portanto, não possuem significado físico, refletindo apenas os erros aleatórios do processo. 
Ressalta-se ainda que, para o equipamento Soxhlet utilizado durante a realização dos experimentos, não foi possível utilizar menores valores de hidromodulo, pois o volume de solvente não era suficiente para ser evaporado e retornar ao balão para nova evaporação. Da mesma forma, também não foi possível aumentar a quantidade de matéria-prima, pois os cartuchos já ocupavam a capacidade máxima do sistema extrator.

A seguir, os dados foram analisados por meio dos diagramas de Pareto no nível de confiança de $95 \%$ e $99 \%$. Contudo, no nível de confiança de $95 \%$ o tempo foi dado como fator significativo, logo, optou-se a prosseguir as análises com o nível de confiança de $99 \%$.

Os efeitos das variáveis independentes do processo considerando o nível de confiança de $99 \%$ estão representados na Figura 2. Pode-se inferir que, o único fator que influencia o sistema é o tamanho da partícula, sendo que o sinal negativo indica que quanto menor o tamanho maior o rendimento. Provavelmente, o fenômeno ocorra devido ao aumento da área superficial efetivamente exposta da matéria-prima com o meio extrativo, neste caso, da casca da cana de açúcar com o hexano, favorecendo o contato e a transferência de massa entre as fases. É importante destacar que, não é possível diminuir o tamanho da partícula indefinidamente, já que chegaremos a um ponto em que ela se tornará indivisível, além do mais, a moagem e a separação adicionam custos ao processo.

As interações das variáveis também não apresentaram efeito significante para o nível de confiança de $99 \%$ e na faixa de estudo, como também observado na Figura 1. Logo, o tamanho da partícula pode ser analisado isoladamente, como já realizado.

A fim de justificar a classificação da casca em diferentes granulometrias fez-se uma extração sem essa etapa, com hidromódulo e tempo dentro da faixa estudada, $10 \mathrm{~g}: 200 \mathrm{~mL}$ e 6hs respectivamente, obtendo-se cerca de $6,5 \%$ de rendimento de cera bruta para um tamanho médio de partícula de $(0,29 \pm 0,06) \mathrm{mm}$. Assim, tem-se que a utilização de granulometrias específicas pode aumentar o rendimento em aproximadamente $54 \%$ para tamanhos de partícula de 0,13mm (Ensaios 1, 2, 5 e 6) e em aproximadamente 153\% para tamanhos de partícula de 0,05mm (Ensaio 15).

Figura 1 - Diagrama de probabilidades dos fatores e de suas interações para o processo de extração da cera da cana de açúcar.

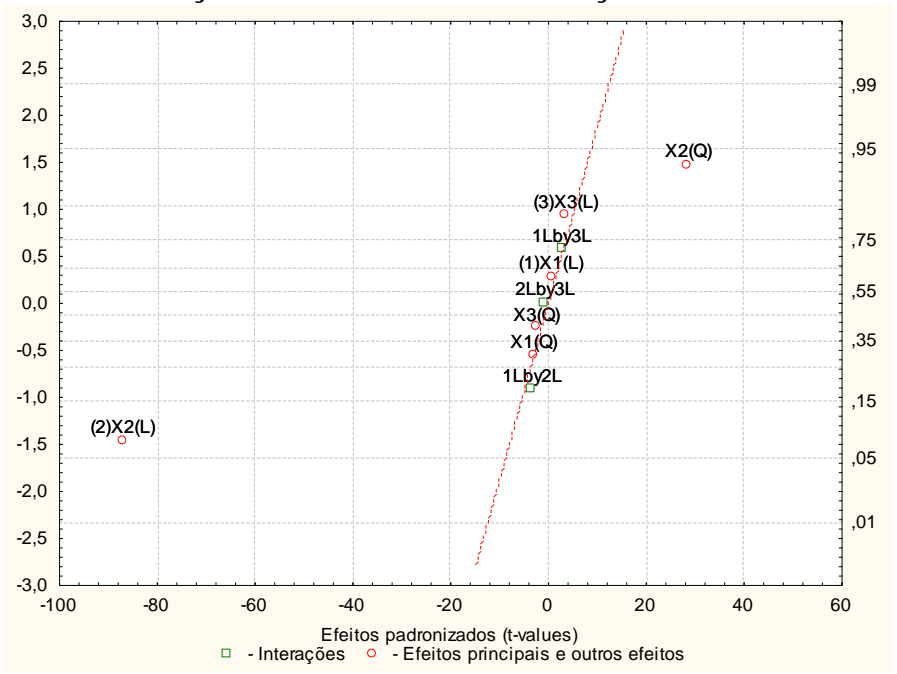


Figura 2 - Diagrama de Pareto dos efeitos sobre a extração da cera da cana de açúcar.

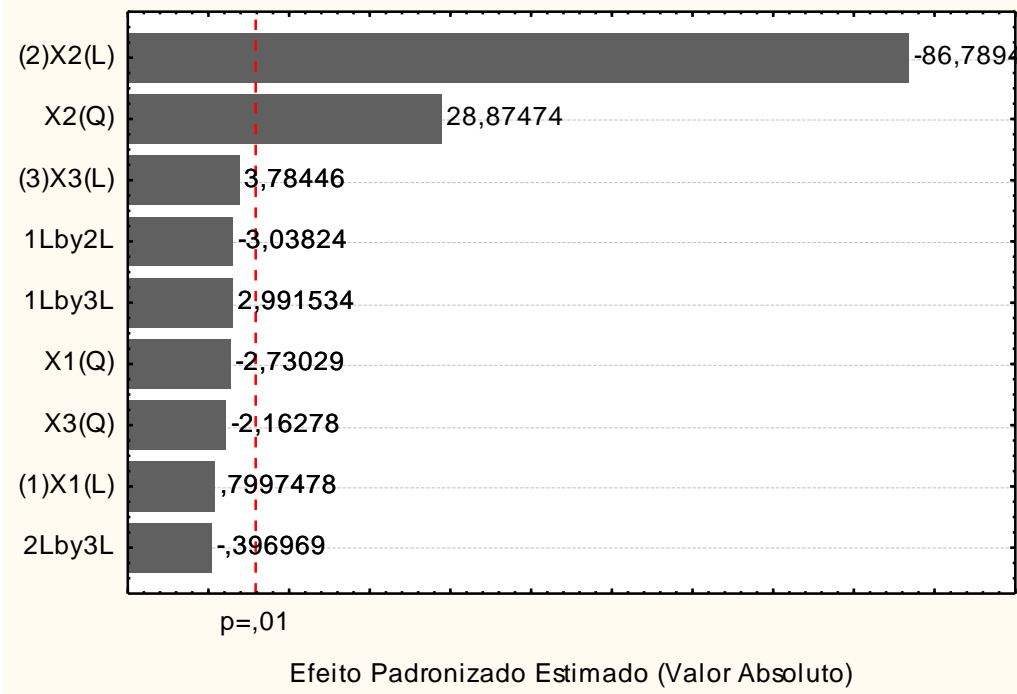

A partir da análise de variância (ANOVA) percebeu-se que, o modelo obtido poderia ser melhorado se as variáveis não significativas fossem ignoradas, assim, considerou-se os coeficientes de regressão apresentados na Tabela 3, cujos respectivos erros foram determinados através da quadruplicata no ponto central, obtendo-se o seguinte modelo matemático quadrático de segunda ordem (Equação 2) para o rendimento do processo:

$$
\mathrm{R}(\%)=6,41755-3,20023 \mathrm{X}_{2}+1,3878 \mathrm{X}_{2}^{2}
$$

em que $\mathrm{X}_{2}$ é o tamanho da partícula em variáveis codificadas.

Tabela 3- Coeficiente de regressão do modelo obtido para o rendimento da extração da cera da cana de açúcar

\begin{tabular}{ccccc}
\hline & Coeficiente & Erro & $\mathbf{- 9 9 \%}$ & $\mathbf{9 9 \%}$ \\
\hline Média & 6,41755 & 0,042607 & 6,16869 & 6,66641 \\
(2) X2 (L) & $-3,20023$ & 0,036874 & $-3,41561$ & $-2,98486$ \\
X2 (Q) & 1,13878 & 0,036899 & 0,92325 & 1,35430 \\
\hline
\end{tabular}

A adequação do modelo acima (Equação 2) também foi avaliada através da análise de variância (ANOVA). Este apresentou um coeficiente de determinação igual a 0,9624, ou seja,

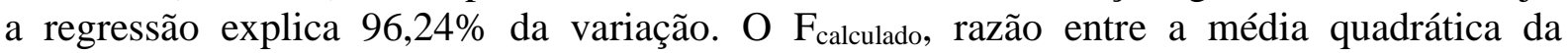
regressão e a média quadrática dos resíduos, é cerca de 30 vezes maior do que o $\mathrm{F}_{\text {tabelado, }}$ considerando o mesmo nível de confiança e graus de liberdade, demonstrando que o modelo se ajusta bem aos resultados obtidos por meio dos ensaios. Outro valor avaliado foi o $\mathrm{F}_{\text {calculado }}$ obtido pela razão entre a média quadrática da falta de ajuste e a média quadrática do erro

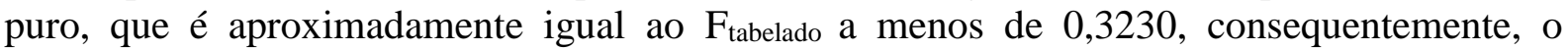
modelo não pode ser utilizado para fins preditivos no nível de confiança de $99 \%$ e na faixa de estudo. Os dados aqui analisados encontram-se na Tabela 4: 
Tabela 4 - ANOVA para o modelo obtido para o rendimento da extração da cera da cana de

\begin{tabular}{ccccccccc}
\hline $\begin{array}{c}\text { Fonte de } \\
\text { variação }\end{array}$ & Soma Quadrática & $\begin{array}{c}\text { Graus de } \\
\text { liberdade }\end{array}$ & $\begin{array}{c}\text { Média } \\
\text { Quadrática }\end{array}$ & Fcalculado & Ftabelado \\
Regressão & 157,5531 & 2 & 78,7766 & $F_{2,15}$ & 191,9881 & $F_{2,15}$ & 6,3589 \\
\hline $\begin{array}{c}\text { Resíduos } \\
\text { Falta de } \\
\text { ajuste }\end{array}$ & 6,1548 & 15 & 0,4103 & & & & \\
\hline $\begin{array}{c}\text { Ejus Puro } \\
\text { Total }\end{array}$ & 6,0991 & 12 & 0,5083 & $F_{12,3}$ & 27,3748 & $F_{12,3}$ & 27,0518 \\
\hline
\end{tabular}

\section{CONCLUSÃO}

A partir deste estudo, nas condições de análise, verificou-se que é possível extrair maiores quantidades de cera quando o tamanho da partícula é diminuído, sendo o nível - $\alpha$ o que apresentou o maior rendimento. Este foi também, o único fator que mostrou-se significativo, indicando que ele pode ser estudado de modo independente para a otimização do processo de extração da cera obtida a partir da casca da cana de açúcar, sem que quaisquer interações com essa variável influencie o sistema. Deste modo, visando uma redução dos custos do processo pode-se trabalhar utilizando menor quantidade de solvente e menor tempo de extração para obter a mesma quantidade de cera. Apesar do modelo matemático obtido não ser preditivo, este se ajusta muito bem aos dados aferidos, isto é, os resíduos deixados pela regressão são mínimos.

\section{REFERÊNCIAS}

BARROS NETO, B. de; SCARMINIO, I. S.; BRUNS, R. E.; Como fazer experimentos: pesquisa e desenvolvimento na ciência e na indústria, 2001.

CONAB 2016 - Companhia Nacional de Abastecimento. Acompanhamento da Safra Brasileira Cana de Açúcar, v. 3 - Safra 2016/17, n.3 - Terceiro levantamento, Brasília, p. 1-74, dez. 2016. ISSN: 2318-7921.

GRANDA, K. M.; Obtenção e caracterização de cera de cana-de-açúcar e suas frações, 2006.

NUISSIER, G., BOURGEOIS, P., GRIGNON-DUBOIS, M., PARDON, P., LESCURE, M.H. Composition of sugarcane waxes in rum factory wastes. Phytochemistry, 61, 2002.

\section{AGRADECIMENTOS} financeiro.

Os autores agradecem à FAPESP (2015/25384-1), SAE, CAPES e CNPq pelo apoio 